\title{
Selection for Improving Field Resistance to Capsicum Chlorosis Virus and Yield-related Traits Using Selection Indices in Peanut Breeding
}

\author{
Jetsada Authrapun ${ }^{1}$, Udomsak Lertsuchatavanich ${ }^{2}$ and Dingming \\ Kang ${ }^{1 *}$ \\ ${ }^{1}$ College of Agronomy and Biotechnology, China Agricultural University, Beijing, \\ China \\ ${ }^{2}$ Department of Plant Pathology, Faculty of Agriculture, Kasetsart University, \\ Bangkok, Thailand \\ *Corresponding Author: Dingming Kang, College of Agronomy and Biotechnology, \\ China Agricultural University, Beijing, China.
}

Received: April 15, 2021

Published: May 11, 2021

(c) All rights are reserved by Dingming Kang., et al.

\begin{abstract}
Capsicum chlorosis virus (CaCV) is a pathogen causing a severe disease in peanut. Therefore, the aim of this study was to select peanut genotypes for improving field resistance to CaCV disease and yield using two selection indices, including a multitrait index based on factor analysis and ideotype-design (FAI-BLUP index) and a multi-trait stability index (MTSI). The 121 genotypes were evaluated under natural infection at the disease hotspots in Thailand from 2017 to 2018, totalling three environments. The experimental design was a randomized complete block with two replications. The 11 traits related to disease resistance and yields such as percentage of disease incidence (DisInc), the area under disease progress curve (AUDPC), pod yield (PY), harvest index (HI), number of pod per plant (PdPt), pod weight per plant (PWPt), seed weight per plant (SWPt), shelling percentage (SH), hundred seed weight (100SW), seed length (SdLgth) and seed width (SdWdth) were collected and used for simultaneous selection. After the selection considering a selection intensity of 15\%, the selection differentials and selection gains obtained by the FAI-BLUP and MTSI indices were positive for almost yield-related traits except for SH and negative for DisInc and AUDPC, which want to be increased and decreased, respectively, indicating that these selection indices provide desirable genetic gains for all traits simultaneously. The genotypes selected by the two selection indices had a good performance for both disease resistance and yield characters. The FAI-BLUP and MTSI indices are efficient method for multi-trait selection and can be used as a tool in selecting promising genotypes based on several targeted traits in plant breeding programs.
\end{abstract}

Keywords: Arachis hypogaea L; Field Resistance; FAI-BLUP Index; Multi-trait Stability Index; Selection Gain

\section{Abbreviations}

DisInc: Percentage of Disease Incidence at 60 Days After Sowing; AUDPC: Area Under Disease Progress Curve; PdPt: Number of Pod Per Plant; PWPt: Pod Weight Per Plant; SWPt: Seed Weight Per Plant; HI: Harvest Index; PY: Pod Yield; 100SW: 100-Seed Weight; SdLgth: Seed Length, SdWdth: Seed Width; BLUP: Best Linear Unbiased Prediction; SD: Selection Differential; SG: Selection Gain

\section{Introduction}

Peanut, also called groundnut (Arachis hypogaea L.) is one of the most economically important legume crops, which provides high quality edible oil and protein. It has been grown worldwide in the tropical and subtropical regions. The goal of peanut breeding is to develop new cultivars with the best combination of desirable traits. 
Increasing pod yield and yield-related traits and improving resistance to biotic and abiotic stresses have been the main objectives in peanut breeding [1]. Therefore, the selection of peanut genotypes based on multiple traits simultaneously is a routine work both in the initial and final stages of the breeding program. Biotic stress due to viral diseases is one of the significant constraints limiting the yield of peanut production [2]. Among pathogenic viruses, Capsicum chlorosis virus (CaCV) has been reported as a cause of disease affecting peanut production in Thailand and the southern part of China and causes significant yield losses [3,4]. CaCV disease is vectored by thrips species [5]. Although the disease is vectored only by thrips, pest management using an insecticide is an ineffective method to control the disease because the thrips vectored can seek refuge in tight spaces of peanut plant [6]. Therefore, genetic improvement for increasing disease resistance and yield traits in peanut breeding is a sustainable method in disease control.

Combining desirable traits in one genotype is a challenge for plant breeders. The selection index is one of the multi-trait selection methods, which can generate the numeric value of genotype for ranking in the selection process [7]. The first selection index used in plant breeding, known as Smith's index, is a linear combination of traits and the optimally economic weight of those traits $[8,9]$. Several selection indices based on Smith's index have been developed and deployed in a breeding program [10]. Most of them require to assign the economic weight of a trait that is difficult to express into the realistic weight due to the lack of a procedure to weigh the trait of economic importance [11]. In addition, the presence of multicollinearity, which occurs when multiple correlated traits are assessed, can cause undermining the effectiveness of traditional selection indices [12]. Recently, the new selection indices that do not require assigning the weight of trait and free from the multicollinearity problem were developed, including a multitrait index based on factor analysis and ideotype-design (FAI-BLUP index) [13] and a multi-trait stability index (MTSI) [14]. The FAIBLUP index is based on a mixed model method and the best linear unbiased prediction (BLUP) to predict the genotypic value for each genotype, which allows ranking the genotypes closer to the genotype-ideotype. The FAI-BLUP index has been applied to select superior genotypes in several crop breeding programs $[15,16]$. Meanwhile, the MTSI is a selection index used for the multi-environment trials (MET) that can combines mean performance and stability of genotype for simultaneous selection based on multiple traits. This method has been used to select the best oat genotypes based on several agronomic and economic traits in analysing multi-environment trials [14]. However, the application of these selection indices has not been reported in peanut breeding.

\section{Objective of the Study}

The objective of this study was to select peanut genotypes for improving field resistance to $\mathrm{CaCV}$ disease along with yield-related traits using the FAI-BLUP and MTSI indices.

\section{Materials and Methods}

Plant material and field experiment

The materials consisted of 121 peanut genotypes, including ICGV86388, a resistant variety to peanut bud necrosis disease (PBND) and thrips developed by the International Crops Research Institute for the Semi-Arid Tropics (ICRISAT), 11 released varieties developed by the Department of Agriculture, Thailand consisting of Kalasin1, Kalasin2, KhonKaen, KhonKaen4, KhonKaen5, KhonKaen6, KhonKaen60-1, KhonKaen60-2, Khonkaen84-7, Khonkaen84-8, and Tainan9, (encoded from V1-V11, respectively) and 109 recombinant inbred lines (RIL) developed by Kasetsart University, Thailand (encoded from 1 to 109). The RILs were originated from the cross Khonkaen5 $\mathrm{x}$ Khonkaen6. The field experiments were conducted in 2017 and 2018 at the hotspot locations of CaCV disease in Kalasin province located in the northeastern region of Thailand, totalling three environments; Somdet district in $2017\left(16.7538^{\circ} \mathrm{N}, 103.7521^{\circ} \mathrm{E}\right)$, Somdet district in 2018 and Mueang Kalasin district in $2018\left(16.4314^{\circ} \mathrm{N}, 103.5059^{\circ} \mathrm{E}\right)$. The experimental design used for each evaluated environment was arranged in randomized completed block, with two replications. The plot size consisted of two rows, with $4 \mathrm{~m}$ long rows comprising of 42 plants/plot. The genotypes were evaluated under natural infection. The susceptible cultivar was grown between the tested genotypes for used as spreader row.

\section{Data collection}

Field resistance to $\mathrm{CaCV}$ disease for each genotype was assessed using a disease incidence rating as described by Puttha., et al. (2008) [17] and the area under the disease progress curve (AUDPC) [18]. At harvesting stage, pod yield (PY, $\mathrm{kgha}^{-1}$ ), harvest index 
(HI), number of pod per plant (PdPt), pod weight per plant (PwPt, g), seed weight per plant (SwPt, g), shelling percentage ( $\mathrm{SH}$ ) and hundred seed weight $(100 \mathrm{SW}, \mathrm{g})$ were collected as describing by Puttha., et al. (2008) [17]. For seed-related traits, the five randomly seeds for each genotype were sampled for the following traits: seed length (SdLgth, inch) and seed width (SdWdth, inch).

\section{Statistical analysis}

The evaluated traits were analyzed for a randomized complete block design using a mixed model following the method proposed by Henderson (1975) [19]. The variance component estimates for each traits were obtained by restricted maximum likelihood (REML) analysis, and the best linear unbiased prediction (BLUP) for each trait and genotype was performed to predict genotypic values. The significance of genotype $(G)$, environment $(E)$ and $G$ $\mathrm{x} E$ interaction effects were tested by a likelihood ratio test. The broad sense heritability) for each trait was calculated based on the variance component estimates. The traits related to field resistance (DisInc and AUDPC) along with yield-related traits (PY, HI, PdPt, PWPt, SWPt, SH, 100SW, SdLgth and SdWdth) were used to select superior genotypes using two selection indices as follows.

A multitrait index based on factor analysis and genotype-ideotype distance (FAI-BLUP)

The BLUP values for each genotype and trait were used to calculate the FAI-BLUP index. This method proposed by Rocha., et al. (2015) [13]. For this study, the maximum values for yield-related traits and minimum values for field resistance parameters were used to identify ideal traits.

\section{A multi-trait stability index (MTSI)}

The MTSI was performed considering mean performance and stability as described by Olivoto., et al. (2019) [14]. The weights of mean performance and stability were assumed to be 50 and $50 \%$ (MTSI-50) and 75 and 25\% (MTSI-75). The selection considering multiple traits was based on the genotype-ideotype distance (Euclidian). The genotype with the lowest MTSI is closer to the ideotype and presents a high mean performance and stability for all traits.

The selection intensity used for each selection index was $15 \%$. After the selection, the selection differential (SD) of all traits were estimated following, where the mean of selected genotypes for the trait and is the population mean of that trait. The selection gain for each trait in percentage (\%SG) was estimated following; , where is the broad-sense heritability. All data analyses and making selection by the two selection index methods were carried out in the $\mathrm{R}$ software with the "metan" package [20].

\section{Results and Discussion}

The genotype effect was significant for almost all traits except for SWPt. The genotype and environment interaction effects were significant for DisInc and 100SW (Table 1). Estimates of variant components for each trait revealed that the contribution of genotypic variance for DisInc and AUDPC were $40.57 \%$ and $44.56 \%$ of phenotypic variance, respectively. For yield-related traits, the contribution of genotypic variance ranged from $5.74 \%$ to 62.7 $\%$ of phenotypic variance (Table 1 ). High values of broad-sense heritability were observed for DisInc, AUDPC, 100SW, SdLgth and SdWdth, indicating a prospect to obtain genetic progress for these traits. Whereas, low to moderate heritability values were observed for PY, PdPt, PWPt, SWPt, HI and SH, ranging from 0.19 to 0.58 (Table 1), demonstrating that these traits are strongly influenced by environmental factors. In this study, the field resistance for each genotype that expresses as reduced disease incidence and minimizes the development of epidemics in the field was assessed using DisInc and AUDPC. Both parameters can effectively differentiate genotypes for field resistance to $\mathrm{CaCV}$ disease. In addition, the AUDPC has been widely used to define field resistance of field crops and can be used as a selection criterion for this trait [17].

In the context of variety testing, the genotype may be considered as fixed or random effects depending on the goal of the analysis, and both are commonly used in practice [21]. In this study, the genotype was assigned to be a random effect; therefore, the best linear unbiased prediction (BLUP) was performed to predict genetic values of genotypes for all accessed traits. Descriptive statistics of the BULPs for each trait are presented in table 2 . The variability of BLUP values for disease resistance and yield-related traits indicated that field resistance to $\mathrm{CaCV}$ disease and yield traits could be improved simultaneously. The application of BLUP in plant breeding has been successfully applied for variety testing, progeny and parent selection and single cross prediction [21]. For peanut, the 


\begin{tabular}{|c|c|c|c|c|c|c|}
\hline \multirow{2}{*}{ Trait } & \multicolumn{2}{|c|}{ Likelihood ratio test } & \multicolumn{2}{|c|}{ Variance components (\%) } & \multirow{2}{*}{$\mathbf{h}^{2}$} \\
\cline { 2 - 6 } & $\mathbf{g}$ & $\mathbf{g ~ x ~ e}$ & $\sigma_{g}^{2}$ & $\sigma_{g x \boldsymbol{~}}^{2}$ & $\sigma_{\boldsymbol{r}}^{2}$ & \\
\hline DisInc & $93.730^{* * *}$ & $9.364^{* *}$ & 40.57 & 10.74 & 48.70 & 0.78 \\
\hline AUDPC & $116.505^{* * *}$ & 2.838 & 44.56 & 5.65 & 49.79 & 0.81 \\
\hline HI & $15.476^{* * *}$ & 0.879 & 16.30 & 5.50 & 78.20 & 0.52 \\
\hline PdPt & $8.657^{* *}$ & 1.339 & 9.55 & 6.35 & 84.09 & 0.37 \\
\hline PWPt & $5.359^{*}$ & 1.883 & 7.47 & 7.67 & 84.85 & 0.31 \\
\hline SWPt & 3.197 & 2.836 & 5.74 & 9.58 & 84.68 & 0.25 \\
\hline SH & $33.781^{* * *}$ & 0.074 & 20.31 & 1.33 & 78.36 & 0.60 \\
\hline $100 S W$ & $81.508^{* * *}$ & $6.279^{*}$ & 36.52 & 9.40 & 54.08 & 0.75 \\
\hline SdLgth & $224.47^{* * *}$ & 1.05 & 62.78 & 2.32 & 35.01 & 0.90 \\
\hline SdWdth & $69.64^{* * *}$ & 1.14 & 31.74 & 4.41 & 63.85 & 0.72 \\
\hline PY & $16.286^{* * *}$ & 0.000 & 12.00 & 0.0 & 88.00 & 0.45 \\
\hline
\end{tabular}

Table 1: Likelihood ratio test, estimated of variant components and heritability for DisInc; percentage of disease incidence, AUDPC: The area under the disease progress curve, PY: Pod yield, HI: Harvest index, PdPt: The number of pod/plant, PWPt and SWPt: Pod and seed weight/plant, SH: Shelling percentage, 100SW: Hundred seed weight, SdLght: Seed length and SdWdth: Seed width evaluated across three environments.

, ,**** significant at $\mathrm{P}<0.05,0.01$ and 0.001 , respectively.

$\sigma^{2}$ : genetic variance; $\sigma_{g x e}^{2}: G x$ E variance; $\sigma_{r}^{2}$ : Residual Variance; $\mathbf{h}^{2}$ : Broad-sense heritability.

\begin{tabular}{|c|c|c|c|c|c|c|}
\hline Trait & Min & Max & Range & $\overline{\mathbf{x}}$ & SD & $\sigma^{2}$ \\
\hline DisInc (\%) & 8.44 & 49.49 & 41.05 & 24.38 & 9.23 & 85.18 \\
\hline AUDPC & 1.02 & 8.86 & 7.84 & 3.62 & 1.67 & 2.79 \\
\hline HI & 0.2476 & 0.3979 & 0.1503 & 0.3326 & 0.0318 & 0.001 \\
\hline PdPt & 13.27 & 18.33 & 5.06 & 15.29 & 1.01 & 1.03 \\
\hline PWPt (g) & 16.02 & 20.87 & 4.85 & 18.22 & 1.08 & 1.17 \\
\hline SWPt (g) & 11.01 & 13.98 & 2.97 & 12.46 & 0.60 & 0.36 \\
\hline SH & 61.82 & 73.14 & 11.32 & 68.65 & 2.62 & 6.89 \\
\hline 100SW (g) & 40.30 & 67.29 & 26.99 & 54.87 & 21.59 & 26.99 \\
\hline SdLght (inch) & 0.469 & 0.748 & 0.279 & 0.588 & 0.052 & 0.003 \\
\hline SdWdth (inch) & 0.331 & 0.407 & 0.076 & 0.370 & 0.013 & 0.0002 \\
\hline PY (kg.ha-1) & 919.5 & 1435.6 & 516.1 & 1165.3 & 105.0 & 11022.8 \\
\hline
\end{tabular}

Table 2: Descriptive statistics of the best linear unbiased predictors (BLUPs) for DisInc; percentage of disease incidence, AUDPC: The area under the disease progress curve, PY: Pod yield, HI: Harvest index, PdPt: The number of pod/plant, PWPt and SWPt: Pod and seed weight/plant, SH: Shelling percentage, 100SW: Hundred seed weight, SdLght: Seed length and SdWdth: Seed width among peanut genotypes evaluated across three environments.

Min: Minimum: Max: Maximum: SD: Standard Deviation: $\sigma^{2}$ : Variance. 
BLUP has been used to progeny and parental selection for disease resistance, yield and quality traits [22-24].

The FAI-BLUP index and MTSI at the two different weights of mean performance and stability were applied to select peanut genotypes for improving field resistance to $\mathrm{CaCV}$ disease and yieldrelated traits based on the disease screening trials at the selection intensity of $15 \%$ using the R solfware package "metan" [20]. Therefore, 18 genotypes were selected in each selection index. For the FAI-BLUP index, the results of the principal component analysis showed that the third main components, which had eigenvalues higher than one, were retained. The cumulated variance in these components was $72.69 \%$ (Table 3), indicating that it is sufficient to represent approximately $73 \%$ of all the variability. The results of factor analysis revealed that the 11 traits were grouped into the three factors (FA) as follow: FA1, PY, HI, PdPt, PWPt and SWPt; FA2,
100SW, SdLgth and SdWdth; and FA3, DisInc, AUDPC and SH (Table 4). These factors were used to design ideotype-genotype. According to the selection objective in this study, maximum BLUP values were desirable for PY, HI, PdPt, PWPt SWPt, SH, 100SW, SdLgth and SdWdth, on the other hand minimum BLUP values were desirable for DisInc and AUDPC. The 18 genotypes selected are shown as in the figure 1 . The selection differentials and selection gains were positive for the yield-related traits that are desired to be positive except for SH and negative for DisInc and AUDPC that want to be decreased (Table 5). The FAI-BLUP index provided total selection gains of approximately $-48 \%$ and $26 \%$ for resistance parameter and yield-related traits that want to be decreased and increased, respectively, indicating that this method provides desirable genetic gains for these traits simultaneously. The FAI-BLUP index has been successfully applied to select superior genotypes in crop breeding programs $[13,15,16]$.

\begin{tabular}{|c|c|c|c|c|c|c|}
\hline \multirow{2}{*}{$\begin{array}{c}\text { Principal } \\
\text { Components }\end{array}$} & \multicolumn{3}{|c|}{ Eigenvalue } & \multicolumn{3}{c|}{ Cumulative variance (\%) } \\
\cline { 2 - 7 } & FAI-BLUP & MTSI-50 & MTSI-75 & FAI-BLUP & MTSI-50 & MTSI-75 \\
\hline PC1 & 3.62 & 3.27 & 3.62 & 32.87 & 29.7 & 32.88 \\
\hline PC2 & 2.51 & 2.05 & 2.16 & 55.71 & 48.4 & 52.49 \\
\hline PC3 & 1.87 & 1.51 & 1.95 & 72.69 & 62.1 & 70.26 \\
\hline PC4 & 0.93 & 1.08 & 0.99 & 81.16 & 71.9 & 79.22 \\
\hline PC5 & 0.79 & 0.87 & 0.82 & 88.38 & 79.8 & 86.73 \\
\hline PC6 & 0.57 & 0.76 & 0.63 & 93.52 & 86.6 & 92.53 \\
\hline PC7 & 0.34 & 0.59 & 0.34 & 96.62 & 92.0 & 95.67 \\
\hline PC8 & 0.20 & 0.42 & 0.23 & 98.43 & 95.9 & 97.83 \\
\hline PC9 & 0.13 & 0.28 & 0.17 & 99.61 & 98.4 & 99.44 \\
\hline PC10 & 0.03 & 0.13 & 0.05 & 99.90 & 99.5 & 99.93 \\
\hline PC11 & 0.01 & 0.05 & 0.01 & 100.0 & 100.0 & 100.0 \\
\hline
\end{tabular}

Table 3: Eigenvalue estimates by principal components analysis and the variance proportion explained by them for the FAI-BLUP and MTSI indices.

MTSI-50 and MTSI-75: multi-trait stability index at the weight of mean performance and stability of 50:50 and 75:25, respectively.

For the MTSI-50 and MTSI-75, the results of principal component analysis revealed that the four-first principal components had eigenvalues higher than one for the MTSI-50, and three-first principal components were observed for the MTSI-75. The cumulative frequency for these principal components for the MTSI-50 and MTSI-75 was $71.9 \%$ and $70.26 \%$, respectively (Table 3). As a result, the 11 traits were grouped into four factors for the MTSI-50 and three factors for the MTSI-75 (Table 4). The genotype selected by the MTSI-50 and MTSI-75 are presented in the figure 2 and 3, respectively. The two MTSI scenarios shared 12 of 18 genotypes 


\begin{tabular}{|c|c|c|c|c|c|c|c|c|c|c|}
\hline \multirow{2}{*}{ Trait } & \multicolumn{3}{|c|}{ FAI-BLUP } & \multicolumn{4}{c|}{ MTSI-50 } & \multicolumn{3}{c|}{ MTSI-75 } \\
\cline { 2 - 13 } & FA1 & FA2 & FA3 & FA1 & FA2 & FA3 & FA4 & FA1 & FA2 & FA3 \\
\hline DisInc & 0.05 & 0.01 & -0.971 & -0.08 & -0.94 & -0.06 & 0.07 & -0.11 & -0.96 & 0.03 \\
\hline AUDPC & 0.03 & 0.00 & -0.97 & -0.01 & -0.93 & -0.11 & 0.03 & -0.07 & -0.95 & 0.07 \\
\hline HI & -0.70 & -0.10 & 0.25 & -0.40 & -0.32 & 0.17 & -0.60 & -0.66 & -0.23 & -0.10 \\
\hline PdPt & -0.83 & 0.27 & -0.05 & -0.88 & -0.03 & -0.11 & 0.07 & -0.84 & 0.04 & 0.22 \\
\hline PWPt & -0.94 & -0.08 & -0.04 & -0.95 & -0.05 & 0.11 & -0.02 & -0.93 & 0.03 & -0.10 \\
\hline SWPt & -0.92 & 0.04 & -0.19 & -0.92 & 0.04 & 0.04 & -0.20 & -0.93 & 0.18 & -0.02 \\
\hline SH & -0.03 & 0.27 & -0.42 & 0.03 & 0.24 & -0.20 & -0.83 & -0.07 & 0.42 & 0.21 \\
\hline PY & -0.81 & -0.15 & 0.11 & -0.51 & -0.28 & 0.34 & -0.27 & -0.79 & -0.12 & -0.18 \\
\hline 100SW & -0.09 & -0.95 & 0.00 & -0.04 & 0.23 & 0.74 & -0.02 & -0.09 & 0.05 & -0.91 \\
\hline SdLgth & 0.12 & -0.78 & 0.06 & 0.05 & -0.04 & 0.71 & 0.22 & 0.11 & -0.11 & -0.78 \\
\hline SdWdth & -0.10 & -0.72 & 0.13 & -0.11 & -0.003 & 0.65 & -0.16 & -0.17 & -0.06 & -0.67 \\
\hline
\end{tabular}

Table 4: Factorial loadings after varimax rotation for DisInc; percentage of disease incidence, AUDPC: The area under the disease progress curve, PY: Pod yield, HI: Harvest index, PdPt: The number of pod/plant, PWPt and SWPt: Pod and seed weight/plant, SH: Shelling percentage, 100SW: Hundred seed weight, SdLght: Seed length and SdWdth: Seed width obtained by the FAI-BLUP and MTSI indices.

${ }^{1}$ Bold values indicate the traits grouped within each factor.

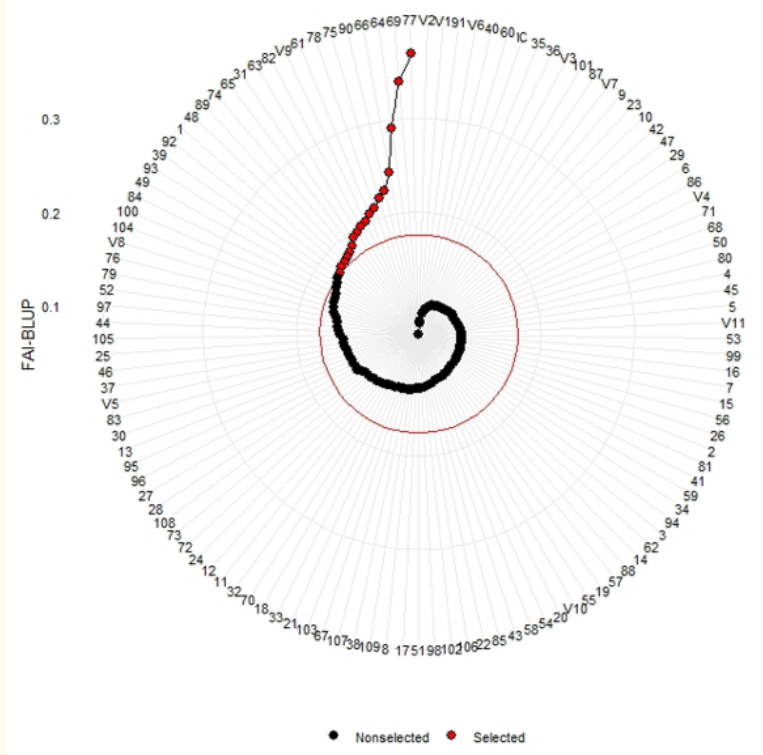

Figure 1: Genotype ranking obtained by the FAI-BLUP index. The selected genotypes are represented in red circle, considering a selection intensity of $15 \%$. including the genotypes $77,78,1,66,63,39,61,82, \mathrm{~V} 8,90,76$ and 92. Meanwhile, the selection differentials and selection gains of the MTSI were positive for almost yield characters except for SH and negative for DisInc and AUDPC. The total selection gains for field resistance parameter and yield-related traits were approximately $-53 \%$ and $49 \%$ for the MTSI-50 and $-52 \%$, and $57 \%$ for the MTSI75 (Table 6). The total selection gains for the resistance parameter obtained by the MTSI at two different weights were close to each other. Interestingly, the total selection gains for yield traits obtained by the MTSI-75 were greater than the MTSI-50. These overall results indicated that the MTSI is an effective method for selecting high-performance and stable genotypes in multi-environment trials based on multiple traits. Furthermore, the weights of mean performance and stability can be adjusted depending on the breeder wants and the purpose of selection in the breeding program and should use several weights to increase the precision and accuracy of selection [14].

The FAI-BLUP, MTSI-50 and MTSI-75 shared eight of 18 selected genotypes, including the genotypes $77,90,78,61,82,63,1$ and 92 (Table 7). Among them, the genotypes 77, 90, 78, 61, 82 and 


\begin{tabular}{|c|c|c|c|c|c|c|}
\hline Trait & Factor & Desirable & BLUP $_{p}$ & BLUP $_{s}$ & SD & $\% S G$ \\
\hline $\mathrm{HI}$ & 1 & Increase & 0.3326 & 0.3755 & 0.043 & 6.71 \\
\hline $\mathrm{PdPt}$ & 1 & Increase & 15.3 & 16.0 & 0.7 & 1.69 \\
\hline PWPt & 1 & Increase & 18.22 & 19.58 & 1.36 & 2.31 \\
\hline SWPt & 1 & Increase & 12.46 & 12.94 & 0.48 & 0.96 \\
\hline PY & 1 & Increase & 1165.3 & 1296.4 & 131.2 & 5.07 \\
\hline $100 \mathrm{SW}$ & 2 & Increase & 54.87 & 58.61 & 3.74 & 5.11 \\
\hline SdLgth & 2 & Increase & 0.5878 & 0.6190 & 0.031 & 4.74 \\
\hline SdWdth & 2 & Increase & 0.3695 & 0.3789 & 0.009 & 1.75 \\
\hline DisInc & 3 & Decrease & 24.38 & 17.50 & -6.87 & -21.98 \\
\hline AUDPC & 3 & Decrease & 3.62 & 2.44 & -1.18 & -26.40 \\
\hline $\mathrm{SH}$ & 3 & Increase & 68.65 & 66.22 & -2.43 & -2.13 \\
\hline \multicolumn{6}{|c|}{ Total (Increase) } & 26.23 \\
\hline \multicolumn{6}{|c|}{ Total (Decrease) } & -48.38 \\
\hline \multicolumn{7}{|c|}{ Genotype selected: 7769646690757861 V9 82633165748948192} \\
\hline
\end{tabular}

Table 5: Estimates of population BLUP mean , BLUP mean of selected genotypes, selection differential (SD) and selection gain (\%SG) for DisInc; percentage of disease incidence, AUDPC: The area under the disease progress curve, PY: Pod yield, HI: Harvest index, PdPt: The number of pod/plant, PWPt and SWPt: Pod and seed weight/plant, SH: Shelling percentage, 100SW: Hundred seed weight, SdLght: Seed length and SdWdth: Seed width obtained by the FAI_BLUP index considering a selection intensity of $15 \%$ and the selected genotypes by each selection index.

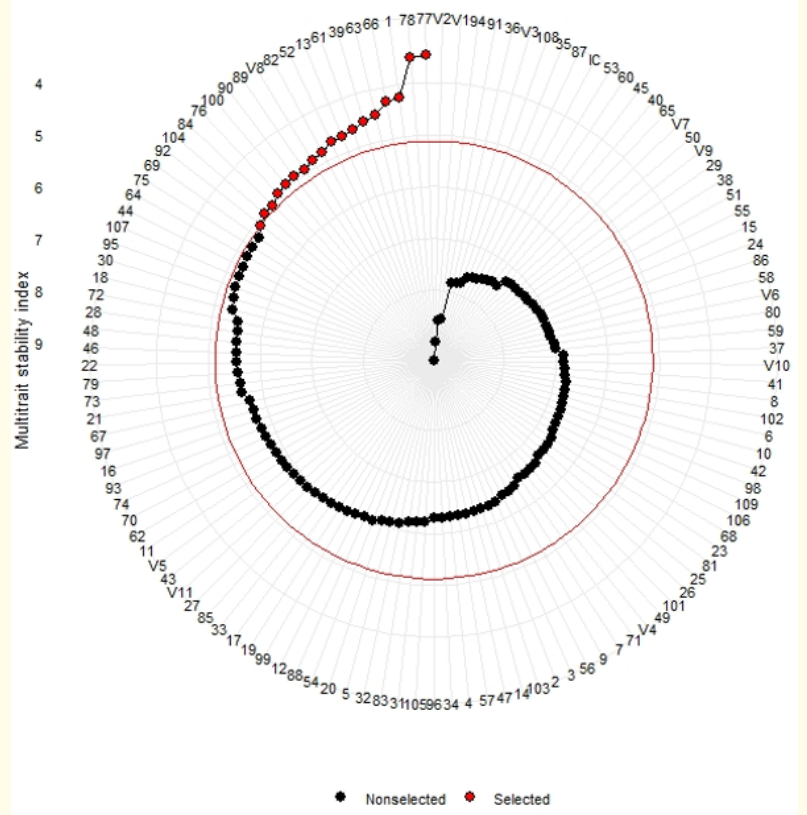

Figure 2: Genotype ranking obtained by the MTSI-50 index. The selected genotypes are represented in red circle, considering a selection intensity of $15 \%$.

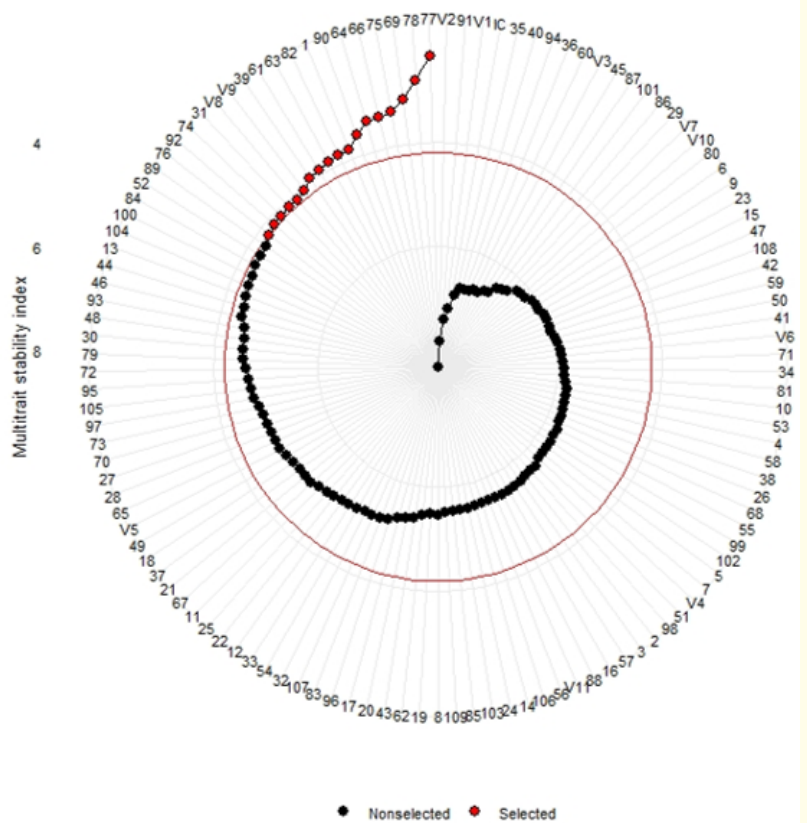

Figure 3: Genotype ranking obtained by the MTSI-75 index. The selected genotypes are represented in the red circle, considering a selection intensity of $15 \%$. 


\begin{tabular}{|c|c|c|c|c|c|c|c|c|c|c|}
\hline \multirow{2}{*}{ Trait } & \multirow{2}{*}{ Desirable } & \multirow{2}{*}{$\mathbf{X}_{\mathbf{P}}$} & \multicolumn{4}{|c|}{ MTSI-50 } & \multicolumn{4}{|c|}{ MTSI-75 } \\
\hline & & & FA & $\mathbf{X}_{\mathrm{s}}$ & SD & $\%$ SG & FA & $\mathbf{X}_{\mathrm{s}}$ & SD & $\% S G$ \\
\hline DisInc & Decrease & 24.47 & 2 & 17.24 & -7.24 & -22.95 & 2 & 16.77 & -7.70 & -24.42 \\
\hline AUDPC & Decrease & 3.64 & 2 & 2.30 & -1.33 & -29.84 & 2 & 2.40 & -1.24 & -27.75 \\
\hline $\mathrm{HI}$ & Increase & 0.3239 & 4 & 0.4075 & 0.084 & 13.50 & 1 & 0.4125 & 0.089 & 14.30 \\
\hline $\mathrm{PdPt}$ & Increase & 15.33 & 1 & 17.0 & 1.7 & 4.04 & 1 & 17.17 & 1.8 & 4.46 \\
\hline PWPt & Increase & 18.27 & 1 & 21.65 & 3.39 & 5.73 & 1 & 22.36 & 4.10 & 6.93 \\
\hline SWPt & Increase & 12.48 & 1 & 14.81 & 2.33 & 4.66 & 1 & 14.42 & 1.94 & 3.87 \\
\hline SH & Increase & 68.58 & 4 & 68.13 & -0.45 & -0.40 & 2 & 65.37 & -3.21 & -2.81 \\
\hline PY & Increase & 1163.0 & 1 & 1461.0 & 298.9 & 11.57 & 1 & 1479.0 & 316.4 & 12.25 \\
\hline $100 \mathrm{SW}$ & Increase & 54.87 & 3 & 59.12 & 4.24 & 5.80 & 3 & 61.18 & 6.31 & 8.62 \\
\hline SdLgth & Increase & 0.5879 & 3 & 0.6022 & 0.014 & 2.4 & 3 & 0.6304 & 0.043 & 6.54 \\
\hline SdWdth & Increase & 0.3696 & 3 & 0.3813 & 0.012 & 3.2 & 3 & 0.3846 & 0.015 & 2.92 \\
\hline \multicolumn{6}{|c|}{ Total (Increase) } & 49.39 & & & & 57.08 \\
\hline \multicolumn{6}{|c|}{ Total (Decrease) } & -52.79 & & & & -52.17 \\
\hline \multicolumn{11}{|c|}{ Genotype selected } \\
\hline \multicolumn{11}{|c|}{ MTSI-50: 7778166633961135282 V8 8990100768410492} \\
\hline \multicolumn{11}{|c|}{ MTSI-75: 77786975666490182636139 V9 V8 31749276} \\
\hline
\end{tabular}

Table 6: Estimates of the population mean , mean of the selected genotypes, selection differential (SD) and selection gain (SG) for DisInc; percentage of disease incidence, AUDPC: The area under the disease progress curve, PY: Pod yield, HI: Harvest index, PdPt: The number of pod/plant, PWPt and SWPt: Pod and seed weight/plant, SH: Shelling percentage, 100SW: Hundred seed weight, SdLght: Seed length and SdWdth: Seed width obtained by the MTSI index considering a selection intensity of $15 \%$ and the selected genotypes by each selection index.

\begin{tabular}{|c|c|c|c|c|c|c|c|c|c|c|c|}
\hline Genotype & $\begin{array}{c}\text { DisInc } \\
(\%)\end{array}$ & AUDPC & HI & PdPt & $\begin{array}{c}\text { PWPt } \\
\text { (g) }\end{array}$ & $\begin{array}{c}\text { SWPt } \\
\text { (g) }\end{array}$ & $\begin{array}{l}\text { SH } \\
\text { (\%) }\end{array}$ & $\begin{array}{c}\text { 100SW } \\
\text { (g) }\end{array}$ & $\begin{array}{l}\text { SdLght } \\
\text { (inch) }\end{array}$ & $\begin{array}{l}\text { SdWdth } \\
\text { (inch) }\end{array}$ & $\begin{array}{c}\text { PY } \\
\left.\text { (kg.ha' }^{-1}\right)\end{array}$ \\
\hline 63 & 11.1 & 1.21 & 0.356 & 15.6 & 18.84 & 12.45 & 64.5 & 58.96 & 0.6347 & 0.3698 & 1306.9 \\
\hline 78 & 11.2 & 1.60 & 0.361 & 15.1 & 18.26 & 12.36 & 66.2 & 63.69 & 0.6415 & 0.4072 & 1242.6 \\
\hline 82 & 11.5 & 1.71 & 0.386 & 15.5 & 19.06 & 12.99 & 68.7 & 56.77 & 0.5850 & 0.3829 & 1257.6 \\
\hline 77 & 17.1 & 2.16 & 0.398 & 16.7 & 19.93 & 12.81 & 62.5 & 64.39 & 0.6466 & 0.3929 & 1407.2 \\
\hline 92 & 24.0 & 3.24 & 0.384 & 16.0 & 19.96 & 13.70 & 70.8 & 55.65 & 0.5924 & 0.3628 & 1270.2 \\
\hline 1 & 29.1 & 4.20 & 0.375 & 16.7 & 19.62 & 13.48 & 70.9 & 57.18 & 0.5975 & 0.3723 & 1316.3 \\
\hline Mean & 24.4 & 3.62 & 0.333 & 15.3 & 18.22 & 12.46 & 68.7 & 54.9 & 0.5878 & 0.3695 & 1165.3 \\
\hline
\end{tabular}

Table 7: The BLUP values for DisInc; percentage of disease incidence, AUDPC; the area under the disease progress curve, PY: Pod yield, HI: Harvest index, PdPt: The number of pod/plant, PWPt and SWPt: Pod and seed weight/plant, SH: Shelling percentage, 100SW: Hundred seed weight, SdLght: Seed length and SdWdth: Seed width of eight shared genotypes selected by the FAI-BLUP and MTSI indices. 
63 had a good performance for both disease resistance and yieldrelated traits, which could be selected for advanced yield trial and used as germplasm in peanut breeding. The advantage of the FAIBLUP and MTSI indices are that both methods are free from the multicollinearity problem among correlated traits, and they do not require assigning the economic weight of trait that is difficult to assign especially for analyzing in the multi-environment trials $[13,14,25]$. Therefore, the two selection indices can solve the main problems of the traditional selection indices in the analysis of the multi-environment trails.

\section{Conclusion}

The simultaneous selection of multiple traits to select promising genotypes is a challenging task for plant breeders. In this study, we applied two selection indices for simultaneous selection based on field resistance to $\mathrm{CaCV}$ disease along with yield characters to select superior peanut genotypes. The results conclude that the FAI-BLUP and MTSI indices are efficient method for multi-trait selection because they provided genetic gains of satisfactory magnitude for several traits simultaneously. Furthermore, the MTSI was considered more appropriate for the analysis of multi-environment trials due to the combination of mean performance and stability for selection. It allows to select the stable genotypes with high mean performance for desirable traits. Therefore, the FAI-BLUP and MTSI indices can effectively be used as a tool in selecting promising genotypes based on targeted traits simultaneously in plant breeding programs. Finally, the application of both selection indices in future studies is to be applied for improving nutritional traits couple with economic characters in peanut breeding.

\section{Bibliography}

1. Pandey KM., et al. "Translational genomics for achieving higher genetic gains in groundnut". Theoretical and Applied Genetics 133 (2020): 1679-1702.

2. Sreenivasulu P., et al. "Virus Diseases of Groundnut". International Institute of Tropical Agriculture, Ibadan, Nigeria (2008): 52.

3. Chen K., et al. "Characterization of a new strain of Capsicum chlorosis virus from peanut (Arachis hypogaea L.) in China". Journal of Phytopathology 155 (2007): 178-181.
4. Chiemsombat P., et al. "Biological and molecular characterization of tospoviruses in Thailand". Archives of Virology 153 (2008): 571-577.

5. Riley D G., et al. "Thrips vectors of Tospoviruses". Journal of Integrated Pest Management 1.2 (2011): 1-10.

6. Srinivasan R., et al. "Resistance to thrips in peanut and implications for management of thrips and thrips-transmitted Orthotospoviruses in peanut". Frontiers in Plant Science 9 (2018): 1604.

7. Acquaah G. "Principles of Plant Genetics and Breeding 2ndedition". Wiley and Sons, Hoboken, NJ, USA (2012): 740.

8. Hazel L N. "The genetic basis for constructing selection indexes". Genetics 28 (1943): 476-490.

9. Smith H F. "A discriminant function for plant selection". Annals of Eugenics 7 (1936): 240-250.

10. Cerón-Rojas JJ and Crossa J. "Linear selection indices in modern plant breeding”. Springer, Cham, Switzerland (2018): 256.

11. Cerón-Rojas JJ., et al. "A selection index method based on eigen analysis". Crop Science 46 (2006): 1711-1721.

12. Prunier J G., et al. "Multicollinearity in spatial genetics: separating the wheat from the chaff using commonality analyses". Molecular Ecology 24 (2015): 263-283.

13. Rocha J R d A S d C., et al. "Multitrait index based on factor analysis and ideotype-design: proposal and application on elephant grass breeding for bioenergy". GCB Bioenergy 10 (2018): 52-60.

14. Olivoto T., et al. "Mean performance and stability in multi-environment trials II: selection based on multiple traits". Agronomy Journal 111 (2019): 1-9.

15. Silva M J., et al. "Evaluation of the potential of lines and hybrids of biomass sorghum". Industrial Crops and Products 125 (2018): 379-385. 
16. Woyann L G., et al. "Multiple-trait selection of soybean for biodiesel production in Brazil". Industrial Crops and Products 140 (2019): 1-7.

17. Puttha R., et al. "Heritability phenotypic and genotypic correlation of peanut bud necrosis virus resistance and agronomic traits in peanut". Asian Journal of Plant Sciences 7 (2008): 276283.

18. Jeger M J and Viljanea-Rollinson S L H. "The use of the area under the disease progress curve (AUDPC) to assess quantitative disease resistance in crop cultivars". Theoretical and Applied Genetics 102 (2001): 32-40.

19. Henderson $C R$ "Best linear unbiased prediction under a selection model". Biometrics 31 (1975): 423-447.

20. Olivoto T and Lúcio AD. "metan: an R package for multi-environment trial analysis". Methods in Ecology and Evolution 11 (2020): 783-789.

21. Piepho H P., et al. "BLUP for phenotypic selection in plant breeding and variety testing”. Euphytica 161 (2008): 209-228.

22. Pattee H E., et al. "Parent selection in breeding for roasted peanut flavor quality”. Peanut Science 28 (2001): 51-58.

23. Pattee H E., et al. "Prediction of parental genetic compatibility to enhance flavor attribute of peanuts". ACS Symposium Series 829 (2002): 217-230.

24. Milla-Lewis S R and Isleib T G. "Best linear unbiased prediction of breeding values for tomato spotted wilt virus (TSWV) incidence in virginia-type peanuts". Peanut Science 32 (2005): 57-67.

25. Burdon R D and Li Y. "Genotype-environment interaction involving site differences in expression of genetic variation along with genotypic rank changes: simulations of economic significance". Tree Genetics and Genomes 15 (2019): 1-10.

\section{Volume 5 Issue 6 June 2021}

(C) All rights are reserved by Dingming Kang, et al. 